\title{
Research on the Difference Mechanism of High Voltage Circuit Breaker's Opening/Closing Coil Current
}

\author{
Yi SU ${ }^{1}$, Yufeng $\mathrm{LU}^{1}$, Lei ZHANG ${ }^{1}$, Xiajin $\mathrm{RAO}^{1}$ \\ ${ }^{1}$ Electric Power Research Institute of Guangxi Power Grid Corporation, Nanning 530023, China;
}

\begin{abstract}
High voltage circuit breakers are important protection and control equipment for the power grid. The defects and faults of the circuit breaker seriously affect the safety and stability of the power system. As the key component of the operating mechanism of the circuit breaker, the opening/closing coils contain a large amount of information about the operating status of the mechanism. In order to investigate the cause of the difference in the current waveforms, this paper analyzes the factors affecting the circuit breaker opening/closing coil current based on the actual high voltage circuit breaker, and distinguishes the characteristics of the current caused by the environmental difference and Structural difference. At the same time, the characteristics of coil current changes under four different types of mechanism defects are analyzed. These results lay the foundation for status evaluation of high voltage circuit breakers based on opening/closing coil current.
\end{abstract}

\section{Introduction}

As one of the most important power equipment in power system, circuit breaker not only plays the role of controlling the load current which is suitable for normal load current, but also plays the protection role of carrying, breaking and closing abnormal current within the specified time. Its reliability not only affects its own safe operation, but also affects the safe operation of other important power equipment in the system 1 . In recent years, a lot of research has been done on the breaker mechanism. The research shows that some mechanism fault information in the circuit breaker operation process will be reflected in some characteristics of the current curve of the opening and closing coil 2. It is of great significance to detect the early defects of the breaker and prevent the fault.

Domestic research on the current of circuit breaker's opening and closing coil mainly focuses on the following aspects. One is to explore the relationship between the opening and closing coil current and the mechanical characteristics of the circuit breaker mechanism. It includes the research on the influence of the change of the operating mechanism's opening and closing coil voltage, opening and closing coil circuit resistance and other parameters on the mechanical performance of the operating mechanism 7; the research on the on-line monitoring method of the mechanism's mechanical state based on the opening and closing coil current 8; the research on the relationship between the circuit breaker's opening and closing coil current waveform and mechanical defects, the extraction of the key characteristic quantity in the coil current waveform, and the establishment of the circuit breaker's normal monitoring system See diagnostic criteria for typical mechanical defects 9. The second is to study the fault of circuit breaker through the current of opening and closing coil. It includes extracting the characteristic value of the current signal of the high-voltage circuit breaker's opening and closing coil, studying the fault identification method of the circuit breaker based on it 10 .

In this paper, based on the existing research, through the fault simulation on the actual circuit breaker, the characteristics of action current waveform are studied. This paper explores the factors that affect the current of the opening and closing coil of the circuit breaker, and compares and analyzes the current characteristics of the opening and closing coil in different fault states. The relationship between fault and current waveform characteristics is established, which lays a foundation for more precise circuit breaker condition assessment through coil current.

\section{Research on Current Waveform Characteristics of Opening and Closing Coils}

\section{1 Operation Principle of Breaker Opening and Closing Coil}

The structure of the opening and closing coil of the breaker is shown in Fig. 1. When the circuit is energized, coil 1 generates electromagnetic force to drive the moving core 3 to move until the moving core 3 collides with the release $\operatorname{rod} 4$, thus triggering the primary opening / closing operation. The circuit of the opening and closing coil is a 
typical inductive circuit, and its basic voltage balance equation is as follows (1).

$$
u=i R+L \frac{d i}{d t}+i v \frac{d L}{d x}
$$

In formula: $\mathrm{u}, \mathrm{I}, \mathrm{R}$ and $\mathrm{L}$ are the voltage, current, resistance and inductance of the coil respectively, $i v \frac{d L}{d x}$ representing the back EMF caused by the movement of the iron core.

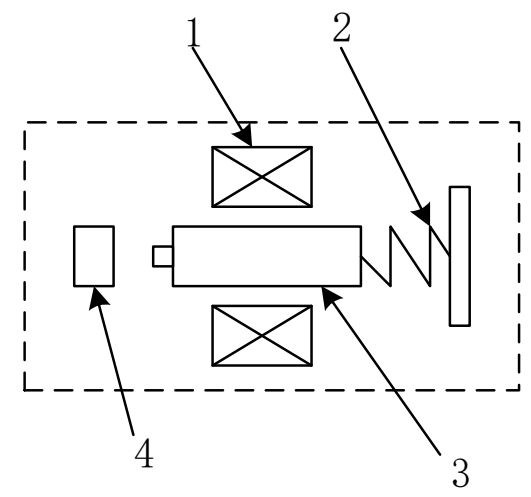

1. Coil 2. Return spring 3. Moving iron core 4. Release lever

Fig. 1 Schematic diagram of circuit breaker's opening/closing electromagnet

Fig. 2 shows the typical current waveform of the opening and closing coil, to is the time when the opening and closing command reaches the circuit on; $\mathrm{t} 1$ is the time when the current in the coil rises to the dominant position of the back EMF; $\mathrm{t} 2$ is the time when the iron core moves to the maximum stroke; $\mathrm{t} 3$ is the time when the time relay is off; $\mathrm{I} 1 \sim \mathrm{I} 3$ are the corresponding current values at $\mathrm{t} 1 \sim$ t3. From to to t1, because the magnetic circuit is not saturated before the coil is electrified, the current increases exponentially at the beginning. When the electromagnetic suction is greater than the reaction force, the iron core starts to move. At this time, the back EMF $i v \frac{d L}{d x}$ in the coil will block the increase of the current. With the continuous increase of the speed of the iron core, the back EMF will also increase, and the current growth rate will slow down. When the speed increases to one At a given time $\mathrm{t} 1$, the dominant current of back EMF begins to decrease to maintain the voltage balance. From t1 to t2, the speed of the core continues to increase and the current decreases obviously until the core moves to the maximum stroke position, and the current reaches the minimum value I2. The speed and amplitude of current decrease are determined by the speed of iron core movement and the parameters of the coil itself. From $\mathrm{t} 2$ to $\mathrm{t} 3$, the core stops moving, the coil current increases and reaches the maximum value $\mathrm{I} 3$ until the circuit is cut off.

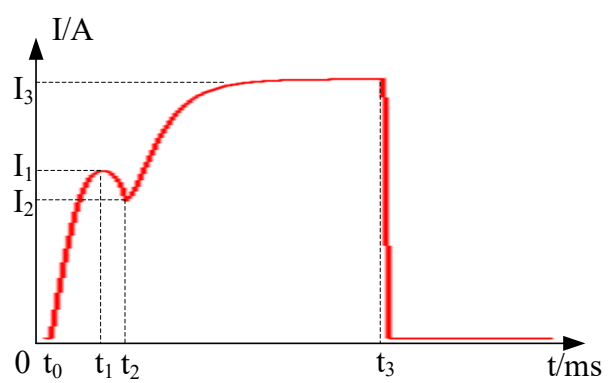

Fig. 2 A typical current waveform of the opening/closing coil

\section{2 Analysis of Influencing Factors on Current of Opening and Closing Coil}

According to the analysis in 1. 1, the current waveform $\mathrm{i}$ is a function of coil voltage $u$, coil inductance $L$, coil resistance $\mathrm{R}$, core movement speed $\mathrm{v}$ and core stroke $\mathrm{x}, \mathrm{i}$. e. $\mathrm{i}=\mathrm{f}(\mathrm{u}, \mathrm{L}, \mathrm{R}, \mathrm{v}, \mathrm{x})$. Therefore, there are differences in coil current waveform, i. e. differences in coil voltage, coil inductance, coil resistance, core movement speed and core stroke. The factors that affect the coil current can be divided into three categories: the first category is environmental parameters, such as ambient temperature; the second category is operating parameters, such as control voltage, loop resistance; the third category is structural parameters of mechanism and coil itself, such as material, geometric size, reaction force, etc.

\section{Analysis of Influencing Factors on Current Waveform of Breaker Opening and Closing Coil under Normal Condition}

\section{1 Influence of Ambient Temperature on Current of Opening and Closing Coil}

In order to investigate the influence of ambient temperature on the current of the opening and closing coil, five A-type coils were put into the constant temperature and humidity test box, and the temperature of the test chamber was adjusted at $0^{\circ} \mathrm{C} \sim 80^{\circ} \mathrm{C}$ and 5 degrees each time. The temperature of the coil is measured by thermocouple. After the coil temperature is stable, the resistance of 5 coils is measured and the average value is obtained. The results of experiment and fitting are shown in Fig. 3.

Coil resistance changes linearly with temperature. In order to further verify the influence of temperature on the current of opening and closing coil, the A-type coil current of $220 \mathrm{kV}$ A circuit breaker is measured under different ambient temperatures, and the results are shown in Fig. 4. The measurement results show that the ambient temperature has a great influence on the current of the onoff coil: the higher the ambient temperature is, the smaller the stable value I 3 of the on-off coil current is, and the corresponding I1 and I2 also decrease in varying degrees. 


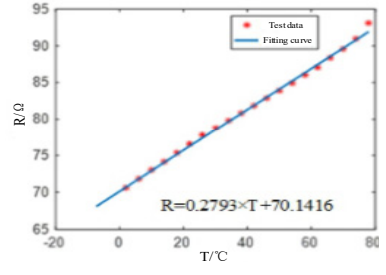

Fig. 3 Variation of coil resistance with temperature

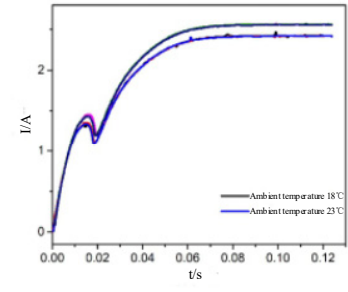

Fig. 4 Coil current at different ambient temperatures

\section{2 Influence of Control Voltage on Current of Opening and Closing Coil}

The change of control voltage of opening and closing coil is a common operating condition. According to the national standard, the control voltage of the opening coil should operate reliably between $65 \% \sim 115 \%(143 \mathrm{~V} \sim$ $253 \mathrm{~V}$ ) of the rated voltage. By adjusting the single-phase voltage regulator, the control voltage can be changed between $65 \% \sim 115 \%(143 \mathrm{~V} \sim 253 \mathrm{~V})$ of the rated voltage of $220 \mathrm{~V}$, and the current waveform of A-type opening and closing coil of $220 \mathrm{kV}$ A circuit breaker is collected under different voltages. The current curve is shown in Fig. 5.

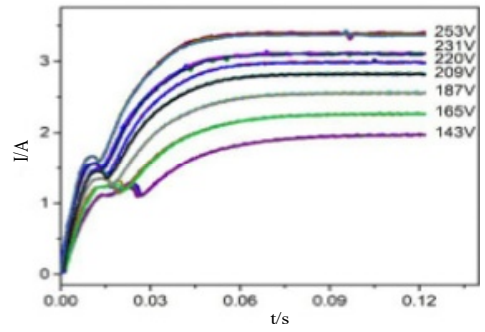

Fig. 5 Coil current at different voltages

The higher the voltage is, the faster the coil current rises in the first stage, and the current I1, I2 and I3 increase in varying degrees. When the voltage is too low, the time of the coil current in the second stage $\mathrm{t} 1 \sim \mathrm{t} 2$ is obviously longer, and even the current waveform is distorted.

\section{3 Influence of Circuit Breaker Mechanism on Current of Opening and Closing Coil}

The closing current waveforms of different circuit breakers of the same type from different manufacturers are randomly tested under the same ambient temperature and rated control voltage. The test results of different manufacturers are shown in Fig. 6, 7 and 8.

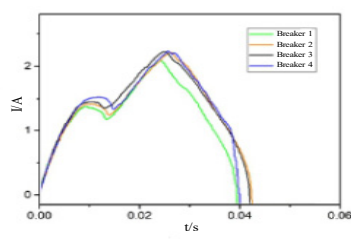

Fig. 6 Comparison of opening current curves of $220 \mathrm{kV}$ A circuit breakers of the same type under normal conditions

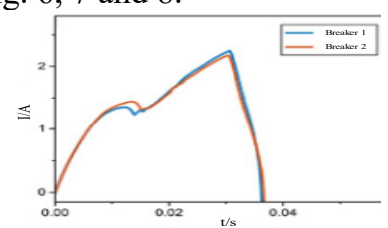

Fig. 7 Comparison of opening current curves of $110 \mathrm{kV}$ B circuit breakers of the same type under normal conditions

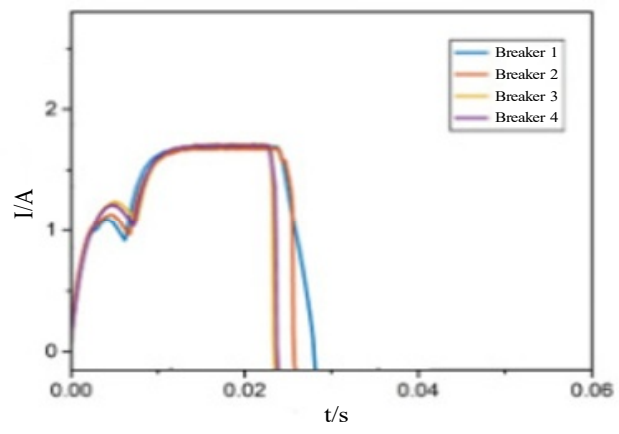

Fig. 8 Comparison of opening current curves of 500kV C circuit breakers of the same type under normal conditions

It can be seen from the test results that under the same external conditions, the current waveforms of the opening and closing coils of different individuals of the same manufacturer and the same type of circuit breaker are quite different, mainly because of the differences caused by the manufacturing dispersion of the circuit breaker body and mechanism structure.

\section{4 The Influence of the Difference of Opening and Closing Coils on the Current}

Four different types of coils are selected, which are type A, type B, type C and type D. Different coils of the same type are tested for closing current on the same mechanism. The control voltage is the rated voltage and the ambient temperature is the same. The typical current waveform is shown in Fig. 9. It can be seen that different individuals of the same type of coil have differences, and there are great differences between different types of coils. The differences of different types of coils are mainly caused by different coil structures and coil materials, while the differences of the same type of coils are mainly caused by the dispersion of coil manufacturing.

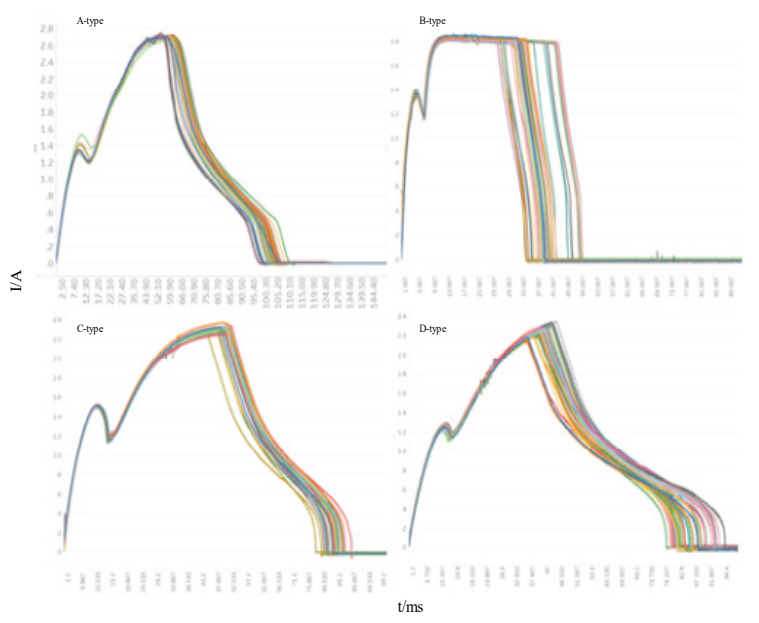

Fig. 9 Current waveforms of different switching coils under the same operating conditions

The above experimental results show that the opening and closing current waveform of each circuit breaker is influenced by the characteristics of the circuit breaker body and has obvious fingerprint characteristics. Therefore, it is necessary to use the opening and closing 
coil current to evaluate the state of the circuit breaker on the basis of eliminating the individual differences of the circuit breaker. The difference of coil current waveform caused by different working conditions can be eliminated by current waveform correction under different working conditions; the difference of current waveform caused by different circuit breakers and different coil structure parameters can be eliminated by the method based on the fingerprint characteristics of circuit breaker individual current waveform; and the difference of current waveform caused by the variation of circuit breaker structure parameters caused by different defects or faults The shape difference is the basis for evaluating the mechanism state of operating circuit breaker.

\section{Analysis of Influencing Factors of Current Waveform in the Case Of Circuit Breaker Opening and Closing Coil Fault}

In order to investigate the influence of breaker defects on the current of the opening and closing coil, the paper uses the fault simulation platform to simulate the various defects of the circuit breaker. In this paper, $220 \mathrm{kV} \mathrm{A}$ circuit breaker is tested on site, and the coil current curve of the circuit breaker under the condition of poor contact, short core clearance, jam of core and short circuit of the opening and closing coil is collected. The current curve of the circuit breaker is on the spot The test platform is shown in Fig. 10.

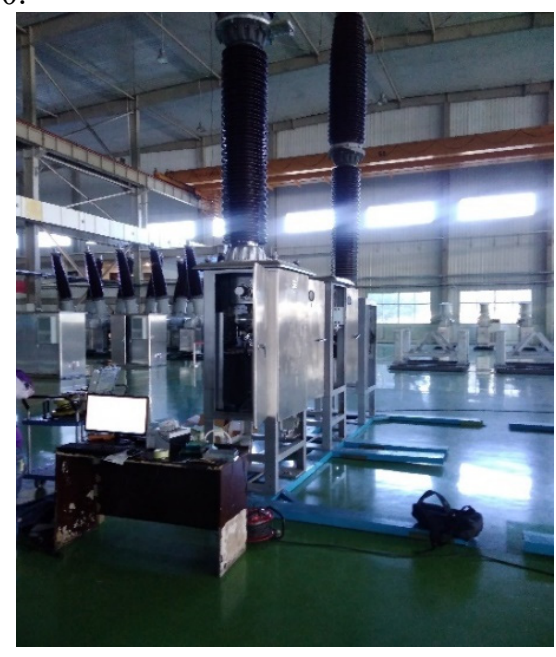

Fig. 10 Test platform

\section{1 Poor Contact of Opening and Closing Coil Circuit}

In the experiment, the actual situation of poor contact is simulated by series resistance. The internal resistance of the closing coil on the actual circuit breaker prototype is $94 \pm 5 \Omega$. In the closing coil circuit, $10 \Omega, 20 \Omega, 30 \Omega, 40$ $\Omega, 50 \Omega, 60 \Omega$ and $70 \Omega$ resistors are respectively connected in series. The coil current during operation is measured, and the results are shown in Fig. 11.

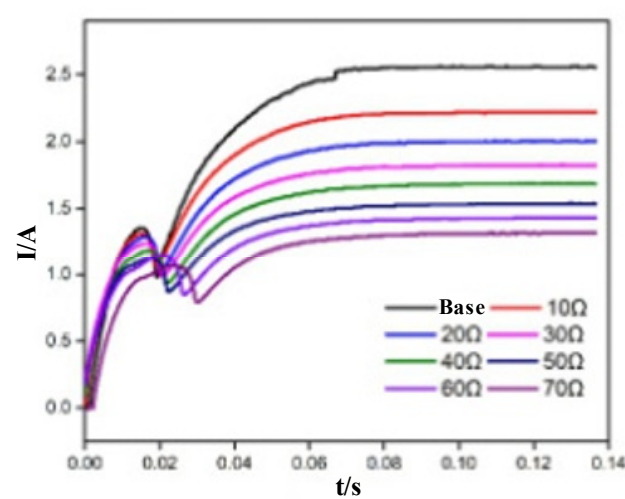

Fig. 11 Coil current waveforms when opening and closing coil circuit is in loose contact

The experimental results show that with the increase of contact resistance, the current in the opening and closing coil changes according to some rules: the first current peak I1 and valley I2 decrease, the stable current I3 decreases, and the peak time $\mathrm{t} 1$ and valley time $\mathrm{t} 2$ increase in varying degrees. At the same time, it should be noted that for the opening coil in the experiment, when the series resistance increases to a certain value, there will be a current fluctuation before reaching the peak I1. The larger the resistance is, the more obvious the fluctuation is, which is related to the movement of the current reducing core. This situation should be considered in the algorithm of evaluating the mechanism state according to the current of the opening and closing coil, so as to avoid misjudgment.

\section{2 The Core Gap Becomes Shorter}

In the actual operation of the circuit breaker, the gap change of the iron core is mostly due to rust, which makes the movement distance of the iron core shorter. Therefore, the gap change of the iron core of the circuit breaker is simulated by adding a copper pad. For the experimental circuit breaker, the normal closing gap is $4 \mathrm{~mm}$, and the gap distances are changed to $3.5 \mathrm{~mm}, 3 \mathrm{~mm}$ and $2.5 \mathrm{~mm}$ respectively through the copper pad (it can not be closed to $2.5 \mathrm{~mm}$ ). The experimental results are shown in Fig. 12.

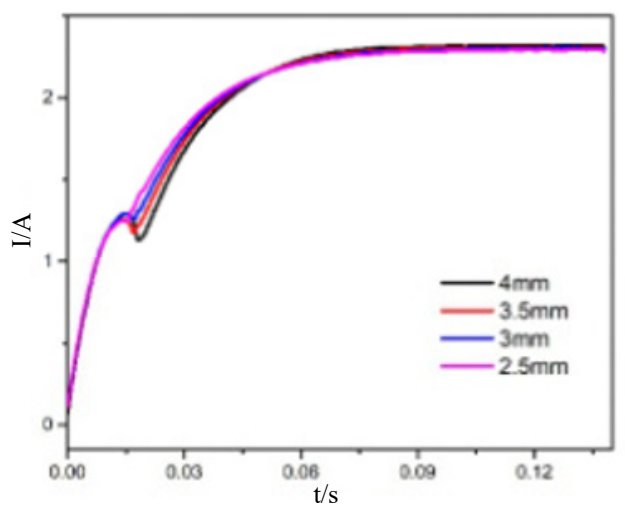

Fig. 12 Coil current waveforms when the core gap decreases

As the core gap becomes shorter, the current waveform changes as follows: the first current peak I1, peak time t1 and stable current I 3 are basically unchanged, the current 
Valley $\mathrm{I} 2$ becomes larger, and the peak time $\mathrm{t} 2$ becomes smaller, that is, I1-I2 and t2-t1 become smaller. When the switch cannot be closed (the gap is $2.5 \mathrm{~mm}$ ), the current curve is close to a straight line. When the gap of iron core becomes smaller, the state of iron core motion is the same at the initial stage, so I1 and 11 are basically unchanged; however, because the distance of acceleration of iron core motion becomes shorter, and the time of touching the top rod becomes shorter, the current Valley 12 becomes larger, the peak time $\mathrm{t} 2$ becomes smaller, and the stable current $\mathrm{I} 3$ is only related to the loop resistance and remains unchanged.

\section{3 Iron Core Jammed}

By changing the spring with different force value, the jam fault of iron core is simulated. Under normal conditions, the force value of the iron core spring of the experimental circuit breaker is $30 \mathrm{n}$. In the experiment, the springs of $60 \mathrm{~N}, 90 \mathrm{~N}, 120 \mathrm{~N}$ and $150 \mathrm{n}$ are replaced respectively. The closing operation is carried out and the current is measured. The results are shown in Fig. 13 and Fig. 14.

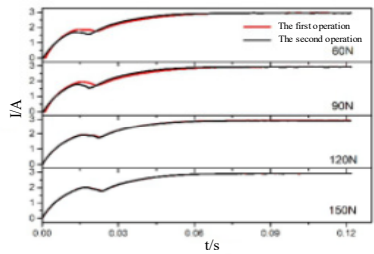

Fig. 13 Coil current waveforms during the first and second action under different pressures

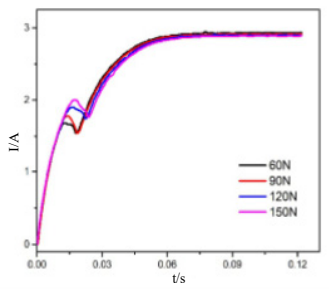

Fig. 14 Coil current waveforms when iron core blocks
It can be seen from the results that when the spring pressure is $60 \mathrm{~N}$ and $90 \mathrm{~N}$, the current of the first action is obviously different from that of the following actions, I1 and $t 1$ are larger, indicating that the resistance of the first action is greater, which proves that the first action of the mechanism is more prone to jam fault after a long time of immobility. When the spring resistance increases to $120 \mathrm{~N}$ and $150 \mathrm{n}$, there is little difference between the first and the following action currents. With the increase of resistance, both the first peak and valley value of current curve and the time to reach the peak and valley value increase, that is, I1, I2, t 1 and $\mathrm{t} 2$ increase, which indicates that the core movement is hindered by jam.

\section{4 Coil Turn to Turn Short Circuit}

The coil inter turn circuit test is conducted by using coils with different turns for defect simulation. The normal coil of circuit breaker is 1900 turns, and the coil of 1700 turns, 1500 turns, 1200 turns and 1000 turns respectively is used for closing operation. The measurement results are shown in Fig. 15.

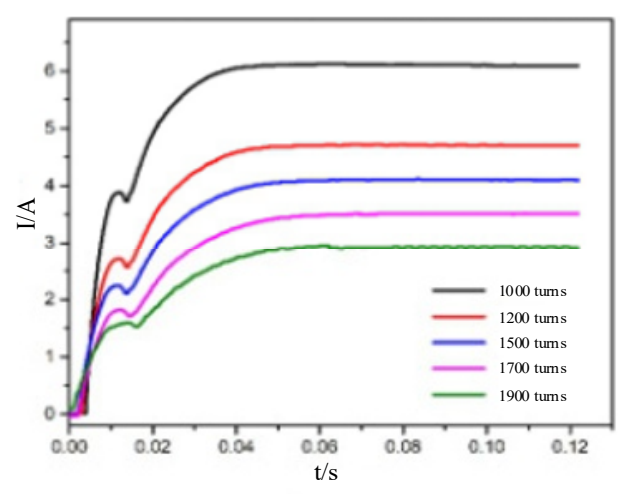

Fig. 15 Coil current waveforms with inter-turn short circuit faults

Although the actual inter turn short circuit not only has the change of the number of gates, but also has the change of inductance and resistance, directly replacing the coil with fewer turns can not completely correspond to the actual situation, but also can reflect the main change of coil current after inter turn short circuit. The experimental results show that with the decrease of coil turns, I1, I2 and I 3 increase, $t 1$ and $t 2$ decrease slightly, and the rising slope of the curve increases obviously.

Through the simulation of the faults and faults in the actual circuit, it is shown that the current characteristics of the opening and closing coils have obvious differences under different defects, and the feasibility of using coil current to locate the fault of the breaker is proved. The results show that the difference between the poor contact fault and undervoltage, turn to turn short circuit and over voltage is very small and difficult to distinguish. In practical engineering application, the circuit undervoltage and over-voltage state can be directly judged by external monitoring means, and no judgment of the opening and closing current is needed. Therefore, the undervoltage and over-voltage faults can be eliminated. The above facts can be located by the current of the opening and closing coil Four faults in the inspection: poor contact, open circuit between turns, jam of core and change of clearance.

To sum up, the key to the condition evaluation of circuit breaker mechanism based on the current waveform of opening and closing lies in two points:

1) The fingerprint characteristics of the circuit breaker are obtained and the influence of non fault factors on the current of the opening and closing coil is eliminated. The main method is to collect the fingerprint data of the opening and closing current waveform under different structure parameters of the circuit breaker by simulation and experimental simulation under different structure parameters of the circuit breaker. Based on the fingerprint data, the environmental parameters, working conditions parameters and the structure parameters of the circuit breaker are modified.

2) The current waveforms of the opening coil in the normal and typical fault states of the circuit breaker are obtained, and the relationship between the type and severity of the defect fault and the variation rate of the current curve is established. 


\section{Conclusion}

As an indicator of circuit breaker mechanism state evaluation, the current of opening and closing coil is easy to obtain and contains rich information. Based on the previous research, this paper further analyzes the difference mechanism of current waveform of opening and closing coil of circuit breaker, and selects typical opening and closing coils of mainstream manufacturers for research. Different environment, working conditions and circuit breaker structure will have a great impact on the current waveform of opening and closing, and the impact of these differences must be eliminated in the condition evaluation of circuit breaker. At the same time, the typical mechanism fault is simulated on the actual circuit breaker, and the relationship between the fault and the change of coil current characteristics is established. Based on this, the key points of condition evaluation of circuit breaker mechanism based on the current waveform of opening and closing are proposed, which is of great significance to the practical application of intelligent condition evaluation of circuit breaker.

\section{Acknowledgement}

This study was supported by the project "Research on Key Technologies of Modular, Intelligent and Standardization Operation Device of $110 \mathrm{kV}$ and above High Voltage Switches (Phase 1: Operation Device for 220kV AIS)" of China Southern Power Grid.

\section{About the author}

1. Yi SU (1988- ), male, Guangxi Province, China, Han nationality, master graduate student, the main research direction for the overvoltage technology.

2. Yufeng LU (1982- ), male, Guangxi Province, China,

3. Lei ZHANG (1986- ), male, Guangxi Province, China, Han nationality, master graduate student, the main research direction for the overvoltage technology.

4. Xiajin RAO (1992- ), female, Guangxi Province, China, Han nationality, master graduate student, the main research direction for the overvoltage technology.

\section{References}

1. XU Guozheng, ZHANG Jierong, QIAN Jiali, et al. Principle and application of high voltage circuit breaker[M]. Beijing: Tsinghua University Press, 2000.

2. CHANG Guang, ZHANG Zhenqian, WANG Yi. Review on mechanical fault diagnosis of high-voltage circuit breakers based on vibration diagnosis[J]. High Voltage Apparatus, 2011, 47(8): 85-90.

3. DE Grijp, M. H. B., Bedet, J. S., et al. Condition monitoring of high voltage circuit breakers[P]. AFRICON, 1996, IEEE AFRICON 4th, 1996.
4. RUNDE R. H. Continuous monitoring of circuit breakers using vibration analysis [J]. IEEE Transaction on Power Delivery 2005, 20(6): 24582465.

5. ZHOU Kaifeng, LI Haitao, XUE Feng, et al. Current characteristic analysis and fault diagnosis of breaking/closing coils in GIS[J]. Electrical Automation, 2017, 39(2): 95-97.

6. ZHANG Yongkui, ZHAO Zhizhong, FENG Xu, et al. Mechanical fault diagnosis of high voltage circuit breakers based on opening/closing coil current parameters[J]. High Voltage Apparatus, 2013, 49(2): $37-42$.

7. Andrusca, M., Adam, M., Pantelimon, Yi, et al. About diagnosis of circuit breakers[P]. 2013.

8. FU Rongrong, ZHAO Lihua, RONG Qiang, et al. Study on Mechanical Characteristics of Operating Mechanism of High Voltage Circuit Breaker [J]. High Voltage Apparatus, 2017, 53(05): 56-62.

9. ZHAO Cheng. On-line monitoring of mechanical characteristics of high voltage circuit breaker[D]. Xi'an; Xi'an Polytechnic University, 2002: 23-26.

10. WANG Yang. The state detection and protection system of circuit breaker off - closing coil[J]. Electronic Test, 2014 (24): 27-29. 